\title{
MÉTAPHORE, POLITESSE DE L'ESPRIT
}

\author{
Daniel Bougnoux ${ }^{1}$
}

Transposons à la communication ce qu'André Breton écrivait du mot comme (préface de Signe ascendant): "Seul le déclic analogique nous passionne: c'est seulement par lui que nous pouvons agir sur le moteur du monde. Le mot le plus exaltant dont nous disposions est le mot COMME, que ce mot soit prononcé ou $t u$. C'est à travers lui que l'imagination humaine donne sa mesure et que se joue le plus haut destin de l'esprit".

L'arc de la métaphore qui met en soudaine relation deux termes, Breton le célèbre pour ses "ressources de fulgurance". De même certaines promesses de communication peuvent faire battre le cœur. Beau comme COMME, beau comme communication..., dirions-nous en parodiant Lautréamont. Et il est de fait qu'une bonne part de la beauté ou de l'esthétique moderne a consisté à jeter des ponts ou à ouvrir les vannes entre des domaines traditionnellement séparés. Fort de ce constat, le poète des Vases communicants prophétisait l' «entrée des médiums». Plus prosaïque, la recherche en sciences de la communication ne semble concernée que par l'entrée des médias; mais la poétique n'en est pas nécessairement absente.

Notre discipline est au carrefour. On dispute encore de ses programmes, de ses limites ou de sa consistance (que beaucoup lui refu-

1 Professeur de Sciences de la communication à l'Université Stendhal de Grenoble-3.

Recherches en communication, $\mathrm{n}^{\circ} 1$ (1994). 
sent). La définir comme interdiscipline ne semble pas une solution sérieuse car l'inter n'a pas de limites, donc de définition claire; or l'université, les collections d'éditeurs, les rubriques de journaux ou les laboratoires de recherche ne fonctionnent bien qu'en se clôturant. Et les enseignements comme les chaînes supposent une grille.

Cette clôture sera d'autant plus nette que la discipline théoricoscientifique reposera sur un noyau dur, c'est-à-dire sur un corpus d'énoncés, d'opérations ou de savoirs non équivoques, et qu'une part croissante du langage vernaculaire s'y laissera formaliser en calcul. A l'autre pôle des connaissances, des disciplines purement verbales demeurent satellisées par les humanités classiques; c'est le cas par excellence de la philosophie, qui consiste moins en un savoir régional qu'en une curiosité, ou une batterie de questions portant sur les conditions, sur la place et les conséquences des savoirs en général. De même aujourd'hui "la communication" exige, pour son enseignement ou sa recherche, qu'on ne l'exerce pas tout à fait comme une spécialité parmi d'autres. Où un étudiant peut-il entendre traiter successivement de l'histoire des ordinateurs, de la dernière campagne de Benetton, de mise en page, de bande dessinée, où peut-il s'initier à la théorie des jeux ou aux négociations d'entreprise? "En communication", et ce n'est pas forcément présomptueux ni incohérent.

Cette chance (ou cet inconvénient) que nous avons sur nos collègues des sciences plus "dures" tient à un certain maniement de la métaphore.

La métaphore est au cœur de la connaissance comme de la relation intersubjective. Il n'y a pas d'effort pour penser qui ne s'en nourrisse. Nous traitons les mots que nous émettons ou que nous recevons comme des éponges ou du caoutchouc, nous tirons dessus pour les déformer, nous les remplissons de notre propre substance et leur prêtons notre vie: c'est ce qu'on appelle faire sens. C'est encore pire avec les images, que personne ne regarde du même œil. Quant aux musiques, aux parfums et aux saveurs, nous savons bien qu'ils sont par excellence le siège de la mémoire affective et qu'ils peuvent héberger des informations incommensurables d'un sujet à l'autre.

Être un sujet, c'est avoir un monde propre, c'est-à-dire habiter un système d'informations, de souvenirs ou d'anticipations qui intègre et oriente à chaque instant tout signal nouveau. Percevoir consiste toujours à interpréter, et adapter sa culture (sa clôture) au monde de l'autre. Dans la conversation comme dans la lecture d'un poème, devant le journal télévisé ou les pubs comme dans la réception d'un 
bon mot, le signal d'une information circule rarement de point à point, mais plutôt de réseau à réseau. Nous dirons qu'une information passe quand telle séquence ou groupage de signaux déclenchera sur le réseau récepteur (que Pierre Lévy propose de concevoir comme un hypertexte) une résonance, un train d'associations, de branchements ou de comportements induits qui traduiront et développeront (plutôt qu'ils ne représenteront adéquatement) la stimulation venue du monde propre de l'autre.

On objectera à cette vision quelque peu solipsiste des résonances ou des réseaux (pensants) le fait, incontestable, de la raison. Il arrive non seulement qu'on s'entende, mais qu'on accorde tous le même sens aux mêmes paroles. Il y a des situations où le cours forcé des mots s'oppose à leur cours flottant.

Ce degré zéro de la métaphore postule une parole transparente, où le signifiant s'efface devant un signifié unique, sans reste ni connotations; où à chaque poste de la communication les sujets parlants maîtrisent la plénitude du concept. Cette parole idéale correspond à l'écriture des contrats, des procès-verbaux, des constats, aux dialogues homme-machines ou au discours en général de la loi: partout où il s'agit de coder sans équivoque une information. C'est le pôle de la valeur économique des messages, donc en particulier celui de la science, qui procède à un élagage extrêmement sévère du sens des mots, mis en lignes, en chaînes ou forcés dans des conduites rectilignes. Mais nous savons que les sciences, qui se font un devoir de tamiser soigneusement leurs concepts, progressent aussi en forgeant de bonnes métaphores. Comparaison n'est pas raison mais "tout se passe comme si...", énonce l'expérimentateur; et voici notre métaphore, chassée par la porte, qui rentre par la fenêtre. Un modèle (un paradigme?), une image peuvent en dire plus qu'un long discours, et donner fortement à penser. En sciences aussi la métaphore est le ressort de la créativité.

A l'autre pôle de nos communications, la poésie concentre traditionnellement les usages métaphoriques du langage. On appelle poétique l'énoncé qui entretient l'équivoque ou une "hésitation prolongée" (Valéry) entre le son et le sens des mots. L'autoréférence ou la circularité souvent remarquées de ces jeux de langage n'abolit pas tout à fait la référence, mais tend à retarder celle-ci, ou à la suspendre. Or la poésie n'a nullement le privilège de la métaphore, et comme on le répète depuis Du Marsais, on sait bien qu'il se fait "plus de figures en une heure à la halle" que dans une journée à l'Académie. La méta- 
phore comme la fonction poétique est éparse, inexpugnable dans la parole et probablement non dénombrable: peut-on énumérer les figures d'un poème? Et combien de métaphores contient cet articleci? Autant compter les chauve-souris sur une planche de Rorschach.

Ces remarques conduisent à penser qu'il est commode mais assez vain d'opposer prose et poésie; et qu'il serait de même illusoire, et peut-être néfaste, de prétendre purger le discours scientifique de toute métaphore. Une des tâches pédagogiques et épistémologiques majeures de notre époque serait au contraire de les réunir, en atténuant la coupure (dans les programmes et dans les têtes) entre la parole littéraire et celle du savant. Nos études de communication pourraient jouer ce rôle, et quelques livres le remplissent déjà: songeons au chefd'œuvre de Douglas Hofstadter, Gödel Escher Bach, qui montre une "même pensée" active dans trois domaines, le théorème de limitation des formalismes mathématiques de Gödel, les boucles étranges et les espaces imbriqués du graveur néerlandais, et les fugues, thèmes, variations et canons du compositeur. Quel est le véritable sujet de cet ouvrage aux mille facettes, sinon la traduction? Comme la pierre de Rosette, le texte de Hofstadter déroule un message en trois langues, entre lesquelles il multiplie les dialogues (entre Achille et la Tortue, entre les chapitres, entre les disciplines, entre l'image et le texte...).

L'un des thèmes récurrents de la polyphonie hofstadterienne est la question de savoir jusqu'à quel point les opérations de l'esprit sont mécanisables, ou modélisables par des machines. L'esprit c'est-à-dire à la fois le mind, le spirit et le witz. La créativité quasi automatique qui s'exprime dans le mot d'esprit semble éloigner absolument celuici du calcul: l'auteur d'un bon mot ne cherche pas, il trouve. De même la "saisie" d'un poème (mais que veut dire saisir, ou plutôt être saisi?) repose sur une connivence ou sur le partage d'une métaphore centrale, qui semble défier jusqu'à un certain point l'explication (car on est toujours libre de ne pas goûter une métaphore, son évidence ne se démontre pas).

Dans son dernier ouvrage, Ma Thémagie, Douglas Hofstadter ne se contente pas de révéler ou d'orchestrer les jeux du "déclic analogique" cher au poète; il conçoit un programme ambitieux de mécanisation de l'analogie ou de la métaphore, "Copycat", et organise autour de lui des tournois d'ordinateurs. On demande par exemple, si $a b c$ devient $x y z$, ce que devient $a b d$ : wyz? xya? xy?... Question de "bonne forme", esthétique donc autant qu'économique, intimement liée au monde propre du sujet connaissant. 
Le postulat de cette recherche est que la créativité commence très "bas" dans l'échelle des performances cognitives; que la métaphore naît avec la moindre de nos perceptions (inséparable de la mémoire, et de l'imagination); et que la modélisation des performances de haut niveau est vaine tant qu' on ne s'attaque pas au tissu subcognitif, donc largement inconscient, de nos liaisons mentales. L'une des leçons de ces prometteuses recherches pourrait être la tolérance. La diversité et la luxuriance infinie des réseaux, en nous et entre nous, s'opposent à l'idée simpliste d'une raison qu' on a pu croire innée, et la même pour tous; connaître, c'est largement reconnaître, et bâtir un modèle du monde avec les ressources de son monde propre. La métaphore irréductible au cœur de la connaissance ne fait jamais qu'exprimer la pluralité et l'incommensurabilité de ces mondes habités par chacun.

La préférence donnée à la métaphore ou à la "fonction poétique" dans la communication enregistre cette diversité, et traduit une politesse énonciative, ou l'aveu lucide qu'il y aura toujours $d u$ jeu entre les partenaires. La métaphore n'est pas faiblesse ni approximation floue, mais ruse et curiosité relancée autour de cette chose énorme, le réel, que nous cadrons, conjurons, contenons, nommons, symbolisons, rêvons ou évitons de mille façons, et qui demeure fièrement inconnu. 